\title{
Anterior Hippocampus and Goal-Directed Spatial Decision Making
}

\author{
Armelle Viard, ${ }^{1,2}$ Christian F. Doeller, ${ }^{1,3,4}$ Tom Hartley, ${ }^{1,5}$ Chris M. Bird, ${ }^{1,3}$ and Neil Burgess ${ }^{1}$ \\ ${ }_{1}^{1}$ UCL Institute of Cognitive Neuroscience, University College London, London WC1N 3AR, United Kingdom, ${ }^{2}$ Inserm-EPHE-UCBN U923, GIP Cyceron, \\ 14074 Caen, France, ${ }^{3}$ UCL Institute of Neurology, University College London, London WC1N 3AR, United Kingdom, ${ }^{4}$ Radboud University Nijmegen, \\ Donders Institute for Brain, Cognition and Behaviour, Centre for Cognitive Neuroimaging 6525, The Netherlands, and ${ }^{5}$ Department of Psychology, \\ University of York, York Y010 5DD, United Kingdom
}

Planning spatial paths through our environment is an important part of everyday life and is supported by a neural system including the hippocampus and prefrontal cortex. Here we investigated the precise functional roles of the components of this system in humans by using fMRI as participants performed a simple goal-directed route-planning task. Participants had to choose the shorter of two routes to a goal in a visual scene that might contain a barrier blocking the most direct route, requiring a detour, or might be obscured by a curtain, requiring memory for the scene. The participant's start position was varied to parametrically manipulate their proximity to the goal and the difference in length of the two routes. Activity in medial prefrontal cortex, precuneus, and left posterior parietal cortex was associated with detour planning, regardless of difficulty, whereas activity in parahippocampal gyrus was associated with remembering the spatial layout of the visual scene. Activity in bilateral anterior hippocampal formation showed a strong increase the closer the start position was to the goal, together with medial prefrontal, medial and posterior parietal cortices. Our results are consistent with computational models in which goal proximity is used to guide subsequent navigation and with the association of anterior hippocampal areas with nonspatial functions such as arousal and reward expectancy. They illustrate how spatial and nonspatial functions combine within the anterior hippocampus, and how these functions interact with parahippocampal, parietal, and prefrontal areas in decision making and mnemonic function.

\section{Introduction}

The neural mechanisms supporting the planning of spatial routes remain a mystery, although single-unit recording and lesion and neuroimaging studies provide some tantalizing hints. Posterior hippocampal formation (HF) in humans (homologous to dorsal $\mathrm{HF}$ in rodents) is thought to encode environmental location to support spatial navigation (O'Keefe and Nadel, 1978; Maguire et al., 1998; Ekstrom et al., 2003; Hartley et al., 2003; Moser and Moser, 1998; Doeller et al., 2008; Moser et al., 2008), whereas anterior HF (homologous to rodent ventral HF) is associated with processing environmental context (Bannerman et al., 2004; Kjelstrup et al., 2008), novelty (Strange et al., 1999; Bunzeck and Düzel, 2006; Dudukovic and Wagner, 2007; Doeller et al., 2008), arousal, emotion, and reward (Moser and Moser, 1998; Ferbinteanu and McDonald, 2001; Fanselow and Dong, 2010; Royer et al., 2010), and their effects on memory (Wittmann et al., 2005; Adcock et al., 2006). Spatial planning links representations of location with expectancy of reward to provide a basis for the appropriate choice of action (Sutton and Barto, 1998; Foster et al., 2000).

\footnotetext{
Received Sept. 3, 2010; revised Dec. 18, 2010; accepted Jan. 24, 2011.

This work was supported by the Wayfinding project of the European Union and the Medical Research Council (UK). We thank Hugo Spiers for useful discussions and help with initial task design, and the Wellcome Trust Centre for Neuroimaging at University College London for providing help and scanning facilities.

Correspondence should be addressed to Neil Burgess, Institute of Cognitive Neuroscience, University College London, 17 Queen Square, London WC1N 3AR, United Kingdom. E-mail: n.burgess@ucl.ac.uk.

DOI:10.1523/JNEUROSCI.4640-10.2011

Copyright $\odot 2011$ the authors $\quad 0270-6474 / 11 / 314613-09 \$ 15.00 / 0$
}

Neuronal responses in rodent hippocampus (Breese et al., 1989; Kobayashi et al., 1997; Frank et al., 2000; Hollup et al., 2001; Fyhn et al., 2002; Ferbinteanu and Shapiro, 2003; Lee et al., 2006; Hok et al., 2007; Dupret et al., 2010) and medial prefrontal cortex (mPFC) (Hok et al., 2005) can reflect the animal's future trajectory or proximity to goal locations. Hippocampal place cells have been proposed to provide spatially extended evaluation functions encoding goal proximity (Burgess et al., 1994; Burgess and O'Keefe, 1996; Bilkey and Clearwater, 2005) or expectancy of reward (Dayan, 1991; Foster et al., 2000). In humans, neuronal representations of location can reflect the current goal of search within a virtual town (Ekstrom et al., 2003), and navigational accuracy is impaired by hippocampal damage (Spiers et al., 2001a,b; Bohbot et al., 2004) and correlates with hippocampal activation (Maguire et al., 1998; Grön et al., 2000; Hartley et al., 2003; Iaria et al., 2003). However, the precise contributions of hippocampal and nonhippocampal regions to spatial decision making remain obscure. For example, although the above rodent studies predominantly target dorsal hippocampus, the spatially extended firing fields in ventral hippocampus (Jung et al., 1994; Kjelstrup et al., 2008) are ideally suited to provide evaluation functions that cover the entire environment.

Using fMRI, we investigated the neural bases of goal-directed spatial planning and the representation of goal proximity. We manipulated memory load to dissociate any general mnemonic role of the hippocampus (Squire, 1992; Eichenbaum et al., 1999) from its role in spatial planning, and to identify regions associated with representation of spatial scenes [e.g., parahippocampal 
gyrus (Epstein and Kanwisher, 1998)]. We also investigated the effect of having to make a detour in order to provide an independent manipulation of difficulty and identify regions associated with inhibition of the direct route [e.g., left and medial prefrontal areas (Maguire et al., 1998; Spiers and Maguire, 2006)]. A key aspect of the design was to parametrically manipulate the start position, hence varying goal proximity.

\section{Materials and Methods}

Participants. Eighteen healthy, right-handed males (mean age $\pm S D, 23.33 \pm 3.96$ years old) with no history of psychiatric or neurological disorder took part in this study, which was approved by the local research ethics committee. All participants gave informed written consent and were paid for participating.

Trial structure. The experiment consisted of 120 trials (24 trials per condition with four experimental and one control conditions; see below). Each trial had an encoding phase and a test phase (lasting $3 \mathrm{~s}$ each) separated by a delay phase (range, 1-7 s; mean, 4 s) (Fig. 1). Trials were separated by an intertrial interval (range, 1-7 s; mean, $4 \mathrm{~s}$ ). In the encoding phase, participants passively viewed a virtual room from a central position; the room contained two parallel walls (with two gaps each), a man behind the second wall, and a gate (supplemental Fig. 1 , available at www.jneurosci.org as supplemental material). The man was the target of mental navigation. From the central position, there were two possible routes to get to the man, corresponding to the two gaps in the first wall. For each condition, there were an equal number of trials with the man on the left or right.

During the test phase, participants were shown the same room as in the encoding image, but from a different start position that varied from trial to trial. There were six start positions, distributed laterally from the middle viewpoint. Participants had to indicate, by pressing one of two buttons, which gap in the nearest wall (left or right) they would choose to get to the man by the shortest possible route. Thus, due to their shifted start position, participants had to mentally recalculate the shortest distance to the man and choose the corresponding gap.

Before scanning, participants were familiarized with the task in a training phase that lasted $\sim 15$ min outside of the scanner, using different start positions than in the experimental task.

Detour and memory manipulation. The experiment used a two-by-two factorial design, the first factor being detour (detour vs no-detour) and the second being memory (memory vs no-memory), resulting in four experimental conditions. These factors concern manipulations of the basic task.

The detour manipulation concerned the gate in the farther wall. In detour trials, the gap in the farther wall directly in front of the target was closed by a gate, meaning that the planned route had to go through the other gap rather than directly to the target. In no-detour trials, the other gap in the farther wall was closed by a gate, meaning that the target could be approached directly (supplemental Fig. 1, available at www.jneurosci.org as supplemental material). Half of the trials required a detour and half were no-detour trials. The distance to the goal was thus longer in the detour condition, although, for the purposes of the planning task (i.e., choosing the closer gap) the goal simply became the farther gap. Accordingly, when comparing the lengths of the alternative routes, we simply measured the distance to the appropriate gap in the farther wall in all cases.

The memory manipulation concerned the test trials. In memory trials, the room beyond the first wall, including the farther wall, gate, and target, was obscured by a curtain during the test phase. Thus, planning required remembered knowledge from the encoding phase regarding the configuration of the farther wall and target, combined with visible knowledge concerning the current start position. Altogether, at test, a curtain was depicted in half of the trials (i.e., memory trials), and in the other half the room remained visible (i.e., no-memory trials).

During control trials (24 trials), one gap (left in half the trials, right in the other half) in the first wall was highlighted and participants simply had to choose that gap, hence controlling for planning processes; it is likely that choosing the highlighted gap demanded less planning than deciding which of two routes was the shortest. Reaction times were indeed shorter for the control condition compared with the experimental conditions.

Trials of the four experimental conditions and the control condition were randomly presented in miniblocks of five trials (unknown to the participants) throughout the experiment, with the constraint that trials of the same condition were not repeated in two successive trials (i.e., across miniblock boundaries).

Parametric variation of start position. For every trial, there were two possible routes to the open gate at the far side of the room (the goal) and one was always shorter than the other. The participants' start position was varied parametrically to manipulate the proximity of the goal (i.e., choosing an obvious short route from a longer route vs choosing between two approximately equal medium-length routes). There were five levels of goal proximity, ranging from distance 1 trials (the shortest correct route to the goal) to distance 5 trials [the longest correct route to the goal and the smallest difference between correct and incorrect routes (Fig. 2)]. All start positions were fully counterbalanced across detour and memory factors. This allowed us to investigate independently the effects of detour, memory, and goal proximity and to establish whether any observed effects could be explained in terms of task difficulty effects. 


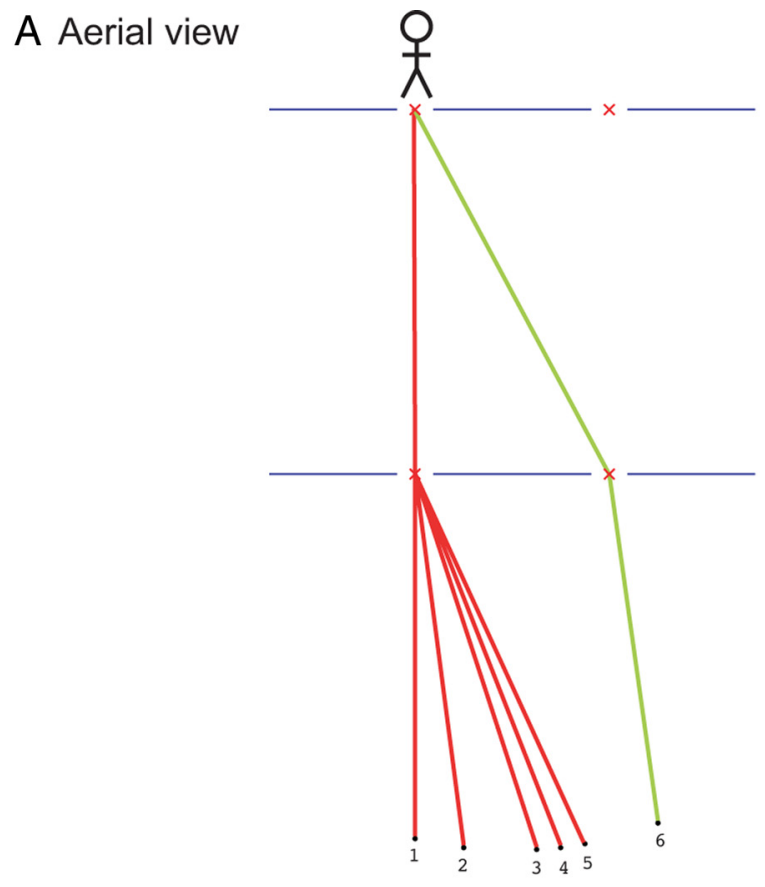

B Start positions at test for the no-detour/no-memory condition Distance 1 Distance 2 Distance 3 Distance 4 Distance 5 Distance 4

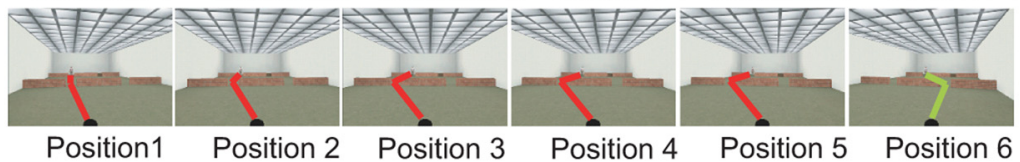

C Start positions at test for the detour/no-memory condition

Distance 1 Distance 2 Distance 3 Distance 4 Distance 5 Distance 4

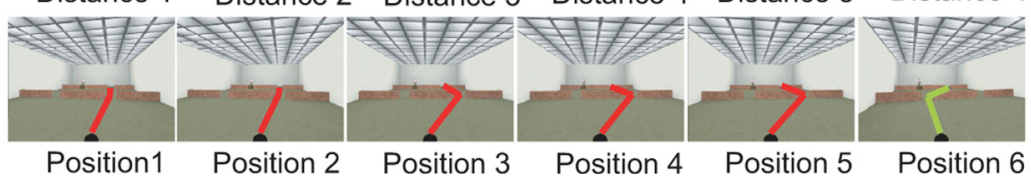

Figure 2. A, Aerial view of the layout, shown in the no-detour condition. $\boldsymbol{B}$, Correspondence between the distance to goal (distances 1-5) and the six start positions (positions 1-6) in the no-detour/no-memory condition, with the shortest route for each start position indicated (participants did not see these routes). In the no-detour condition, the shortest route for start positions 1-5 (red) involved choosing the gap opposite to the man, whereas the shortest route from the sixth start position (green) involved choosing of the gap on the other side. C, Correspondence between distance to goal and start positions in the detour/no-memory condition, with the shortest route for each start position indicated. In the detour condition, the brown gate in the far wall blocks the direct route to the man, so that the goal for the planning task (i.e., the choice of gate in the nearer wall) becomes the other (unblocked) gate in the farther wall. The start positions are organized relative to the goal for the planning task (i.e., number 1 is the closest to the goal in all conditions, but is far from the man in the detour condition).

Overall, in $83 \%$ of test trials (start positions $1-5$ ), the shortest route from the test viewpoint (i.e., the choice of left or right gap) was the same as from the encoding viewpoint. For the remaining 17\% of trials (start position 6), the shortest route was through the other gap in the nearest wall compared with that shown at encoding. Using this shortest route, start position 6 was the same distance from the goal as start position 4 (Fig. 2).

Behavioral data analysis. A repeated-measure ANOVA was applied to the experimental factors [detour $(2) \times$ memory $(2) \times$ goal proximity (5)] for both performances and reaction times (RT). Performance was calculated by dividing the number of correct trials by the total number of trials for that configuration (hit rate).

fMRI acquisition. Blood oxygenation level-dependent-sensitive T2*weighted functional images were acquired on a 3T Siemens Allegra scanner using a gradient-echo EPI pulse sequence with the following parameters: repetition time, $2600 \mathrm{~ms}$; echo time, $30 \mathrm{~ms}$; flip angle, $90^{\circ}$; slice thickness, $2 \mathrm{~mm}$; interslice gap, $1 \mathrm{~mm}$; in-plane resolution, $3 \times 3$ $\mathrm{mm}$; field of view, $192 \mathrm{~mm}^{2}$; 40 slices/volume. The sequence was opti- mized to minimize signal dropouts in the medial temporal lobes (Weiskopf et al., 2006). Visual stimuli were presented using MATLAB (MathWorks) and COGENT 2000 Toolbox (http://www.vislab.ucl.ac.uk/cogent.php).

Image preprocessing. Functional images were processed and analyzed using SPM5 (www.fil. ion.ucl.ac.uk/spm). The first five volumes were discarded to allow for T1 equilibration. Standard preprocessing procedures included correction for differences in slice acquisition timing, realignment/unwarping to correct for interscan movements, and normalization of the images to an EPI template specific to our sequence and scanner that was aligned to the T1 MNI template. Finally, the normalized functional images were spatially smoothed with an isotropic $8 \mathrm{~mm}$ FWHM Gaussian kernel.

$f M R I$ data analysis. In the basic model, fMRI time series were modeled by a general linear model including separate regressors for each of the four conditions during the test phase (nodetour/no-memory,no-detour/memory, detour/ no-memory, and detour/memory) and for the control condition, as well as parametric modulations of these regressors reflecting the goal proximity in each trial. In addition, we modeled the encoding phase by including one regressor containing all trials. The resulting coefficients were analyzed across participants using SPM5. All regressors were convolved with the canonical hemodynamic response function. Data were high-pass filtered (cutoff period, $128 \mathrm{~s}$ ). Coefficients for each regressor were estimated for each participant using maximum likelihood estimates to account for serial correlations in the data. At the first level, linear contrasts of the parameter estimates for each regressor of interest were calculated for each participant and were then brought to the second level random-effects analysis. We conducted a $2 \times 2$ ANOVA with the factors detour and memory on the parameter estimates for the regressors of the four experimental conditions at test.

To look at the parametric effect of goal proximity, we extended the above statistical model by introducing parametric regressors reflecting differences in goal proximity on a trial-by-trial level. This design enables us to look at any possible effects of goal proximity per se, but also any possible effects of detour and memory on goal proximity and vice versa. To control for possible confounds due to response-switching (i.e., changing from the gate that would have been chosen from the encoding viewpoint) and novelty (i.e., the change in the visual scene from the encoding to testing viewpoints) on the goal proximity effect, we estimated a second first-level model. This model included six regressors according to the six different starting positions (collapsed across the four experimental conditions), plus one separate regressor for the control condition and an additional regressor modeling activity in the encoding phase (collapsed across all conditions; see above).

A final analysis was performed to investigate whether activations identified as reflecting goal proximity could be explained in terms of a nonspecific task-difficulty effect. We therefore estimated another first-level model that included a regressor for the experimental conditions (collapsed across all four conditions), a regressor for the control condition, and another for the encoding phase. For the first regressor, two parametric modulations were entered, reflecting goal proximity (as defined in 
A Performance (\%)

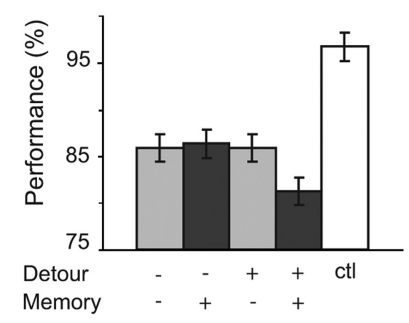

B Reaction times

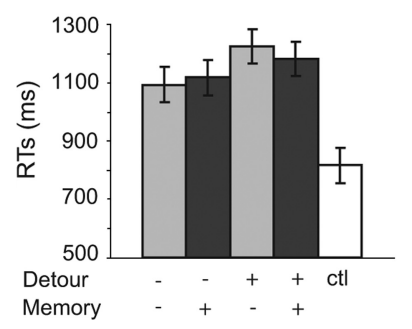

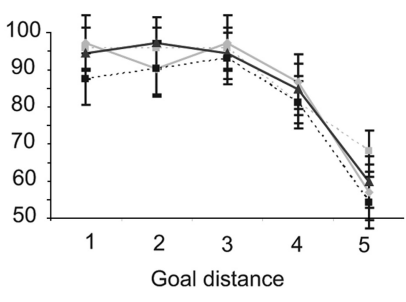

NoD/NoM NoD/M $\overrightarrow{\mathrm{D} / \text { NoM }} \quad \overrightarrow{\mathrm{D} / \mathrm{M}}$

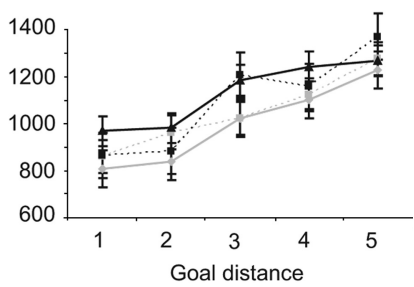

Figure 3. $A, B$, Behavioral results for the detour, memory, and control conditions in terms of performance (i.e., choice of the correct shortest path) $(\boldsymbol{A})$ and reaction times ( $\boldsymbol{B}$, left). The effect of goal proximity ( $\boldsymbol{B}$, right) (for distance levels, see Fig. 2) on performance and reaction times is shown separately for the detour and memory conditions. Error bars denote SEM. D, Detour; M, memory; NoD, no-detour; NoM, no-memory; ctl, control.

Parametric variation of start position, above) and task difficulty (as reflected in RTs on a trial-by-trial level). The corresponding parameter estimates were calculated for each participant and brought to the secondlevel random-effects analysis (one-sample $t$ tests). Each parametric analysis therefore revealed the unique contribution of either goal proximity or task difficulty, since each was orthogonalized to the rest of the design.

Based on our a priori hypotheses, we report activations at an uncorrected statistical threshold of $p<0.001$ and extent threshold of $k>10$ voxels. Coordinates of brain regions are reported in MNI space.

\section{Results}

\section{Behavioral results}

Analyses of the behavioral data revealed a significant interaction between the factors detour and memory for performance (Fig. 3), indicating that performance decreased when having to make a detour on the basis of remembered information $\left(F_{(1,17)}=6.514\right.$, $p<0.021$ ). RTs showed a main (increasing) effect of having to make a detour $\left(F_{(1,17)}=17.976, p=0.006\right)$. A main effect of goal proximity was also detected, both in terms of performance increasing with goal proximity $\left(F_{(4,13)}=24.089, p<0.001\right)$ and $\operatorname{RTs}\left(F_{(4,13)}=20.142, p<0.0001\right)$ and RTs decreasing with goal proximity. Performance showed no sign of an interaction between goal proximity and detour $\left(F_{(4,13)}=0.512, p=0.727\right)$, nor between goal proximity and memory $\left(F_{(4,13)}=0.616, p=0.652\right)$, nor a triple interaction $\left(F_{(1,13)}=1.390, p=0.247\right)$. Similarly, RTs showed no interaction between goal proximity and detour $\left(F_{(4,13)}=\right.$ $1.107, p=0.361)$, nor goal proximity and memory $\left(F_{(4,13)}=0.942\right.$, $p=0.445)$, nor a triple interaction $\left(F_{(4,13)}=1.761, p=0.147\right)$. Thus, varying goal proximity affected performance/difficulty independently of the detour and memory manipulations. Furthermore, all subjects completed the task successfully with $90 \%$ (SD, $6 \%$ ) of correct responses.

\section{fMRI results}

Spatial planning

Comparing all experimental conditions (planning a route) to the control task (no spatial planning) showed activation in a fronto-

Table 1. Results of the contrast comparing all conditions to the control condition (highlighted gate)

\begin{tabular}{llrrrr}
\hline & & \multicolumn{3}{c}{ MNI coordinates } & \\
\cline { 3 - 5 } Region (BA) & Laterality & \multicolumn{1}{c}{$y$} & \multicolumn{1}{c}{$z$} & Zscore \\
\hline Medial PFC (6) & $\mathrm{R}$ & 28 & 0 & 54 & 6.08 \\
& $\mathrm{~L}$ & -24 & -2 & 64 & 5.42 \\
Dorsolateral PFC (6, 46/9) & $\mathrm{L}$ & -24 & 0 & 56 & 5.39 \\
& $\mathrm{R}$ & 46 & 44 & 20 & 4.68 \\
Ventrolateral PFC (44) & $\mathrm{R}$ & 62 & 14 & 14 & 4.52 \\
& $\mathrm{~L}$ & -48 & 28 & 28 & 3.62 \\
Parahippocampal gyrus (36) & $\mathrm{R}$ & 24 & -38 & -10 & 3.28 \\
Precentral gyrus (9) & $\mathrm{L}$ & -52 & 6 & 40 & 3.89 \\
Precuneus (7) & $\mathrm{L}$ & -4 & -64 & 60 & 5.53 \\
& $\mathrm{R}$ & 6 & -42 & 50 & 5.10 \\
PPC (40) & $\mathrm{L}$ & -34 & -42 & 44 & 4.88 \\
& $\mathrm{R}$ & 36 & -50 & 42 & 4.39 \\
ACC (32) & $\mathrm{R}$ & 6 & 24 & 34 & 4.42 \\
Insula & $\mathrm{R}$ & 34 & 26 & -4 & 4.83 \\
& $\mathrm{~L}$ & -38 & 16 & 8 & 4.07 \\
Middle occipital gyrus (19) & $\mathrm{R}$ & 36 & -76 & 36 & 5.27 \\
Lingual gyrus (19) & $\mathrm{R}$ & 16 & -40 & -4 & 3.39 \\
Thalamus & $\mathrm{R}$ & 8 & -8 & 4 & 3.46 \\
Cerebellum & $\mathrm{L}$ & -14 & -56 & -50 & 4.59 \\
& $\mathrm{~L}$ & -6 & -76 & -26 & 4.21
\end{tabular}

$Z$ scores are for clusters of more than 10 voxels at $p<0.001$. ACC, Anterior cingulate cortex; BA, Brodmann area(s); $\mathrm{R}$, right; L, left.

parietal network, including the bilateral $\mathrm{mPFC}$, dorsolateral and ventrolateral prefrontal cortex (PFC), posterior parietal cortex (PPC), precuneus, insula, right parahippocampal gyrus, occipital regions, thalamus, and cerebellum (Table 1).

\section{Effect of detour}

Compared with the no-detour trials, the detour trials showed greater activation in the left $\mathrm{mPFC}$ and right ventromedial PFC, bilateral precuneus, left PPC, and right cerebellum (Fig. $4 A$, Table 2). Different areas were activated by goal proximity (see Parametric effects of goal proximity, below), indicating that detour-related activations did not simply reflect goal proximity or difficulty. Right anterior hippocampal activation (and left activation, subthreshold at $p=0.005)$ was detected when contrasting the no-detour (i.e., direct route) to the detour trials (i.e., longer, indirect route). We interpret this activation as reflecting proximity to goal (see Parametric effects of global proximity, below).

\section{Effect of memory (obscuring the scene at test)}

When participants had to choose the route based on their memory for the layout of the scene (curtain vs no curtain trials), there was greater activation bilaterally in the parahippocampal and lingual gyri (Fig. $4 B$, Table 2). The reverse contrast (no curtain $>$ curtain trials) revealed bilateral inferior occipital activation.

\section{Detour and memory interaction}

An interaction effect was found in the left hippocampus, bilateral ventrolateral and ventromedial PFC, and medial and posterior parietal areas. A weak interaction can be seen in bilateral anterior hippocampus (Fig. $4 A$ ), such that the effect of memory is greater in no-detour trials than in detour trials (in which planning may overshadow memory). This interaction becomes significant in a small, left mid-hippocampal region and in the other regions listed above, where the memory effect reverses in detour trials (i.e., greater activation in the no-memory condition in which the goal is visible). It is possible that the visible detour trial evokes different or more intense spatial planning than other conditions, but this interaction was not anticipated and its interpretation is speculative. 
A Detour effect
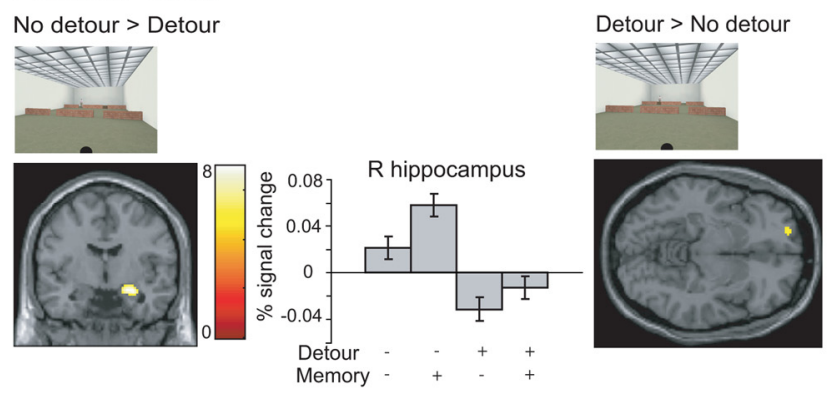

\section{B Memory effect}
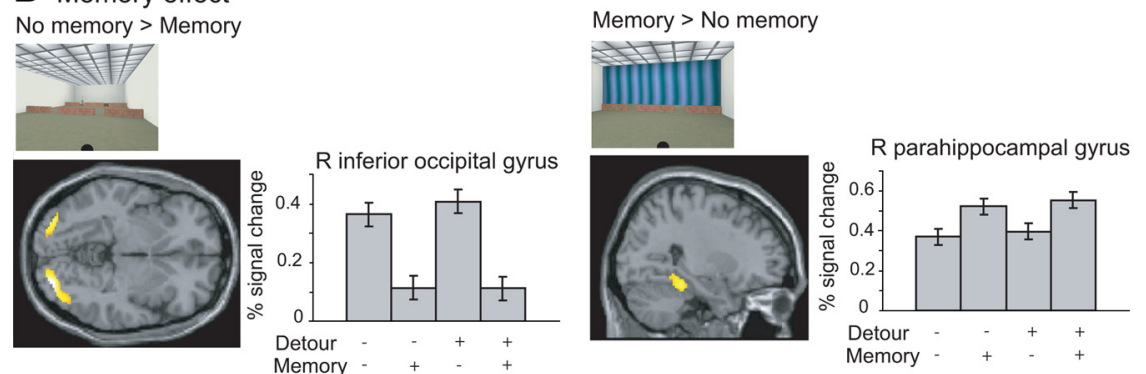

Figure 4. Statistical parametric maps showing the main results of the factorial analysis. $\boldsymbol{A}$, Main effect of detour: no-detour versus detour, centered on the right hippocampal peak $(x=26, y=-6, z=-16)$; detour versus no-detour, centered on the left mPFC peak (Brodmann area 10; $x=-18, y=58, z=-8$ ). B, Main effect of memory: no-memory versus memory, centered on the right inferior occipital peak (Brodmann area 18; $x=30, y=-88, z=-4$ ); memory versus no-memory, centered on the right parahippocampal peak (Brodmann area $36 ; x=28, y=-38, z=-18$ ). Stereotaxic coordinates are given in Table 2. Plots show mean percentage signal change of the peak voxel in the indicated region ( \pm SEM) separately for the detour and memory factors. Color scale shows voxel $t$ values. L, Left; R, right.

Parametric effects of goal proximity

We first investigated which regions showed levels of activation that increased parametrically the closer the start location was to the goal. This analysis revealed activations in bilateral anterior hippocampus (peak region). In addition, we observed a network of regions, including right parahippocampal gyrus, bilateral ventromedial PFC, mPFC, and bilateral posterior cingulate gyrus, extending into the precuneus and retrosplenial cortex, occipital areas (lingual gyrus, cuneus), right anterior cingulate, and superior temporal cortices and bilateral cerebellum (Fig. 5, Table 3). This parametric effect is independent of detour and memory factors since we did not observe significant main effects of detour or memory in the aforementioned regions in the $2 \times 2$ ANOVA of these effects on the parametric estimates for goal proximity (i.e., goal proximity did not interact with detour or memory in its effect on activation) (see Materials and Methods). Calculation of the full distance to the goal (i.e., the distance to the goal location itself, rather that the distance to the open gate in the rear wall) led to similar results. Indeed, the increased hippocampal activation for no-detour trials compared with detour trials (i.e., shorter vs longer overall routes to the goal) (Fig. 4) is consistent with our main finding that hippocampal activation is driven by goal proximity.

To further rule out the possibility that the goal proximity effect could be explained by the varying difficulty of the task, an additional parametric analysis was conducted to reveal the unique contribution of goal proximity (i.e., the design matrix included orthogonalized regressors for goal proximity and for task difficulty, as assessed by RTs on a trial-by-trial level). Results showed that the goal proximity-related activations persisted in the same regions when difficulty was taken into account, namely the bilateral hippocampus, parahippocampal gyrus, $\mathrm{mPFC}$, pos-

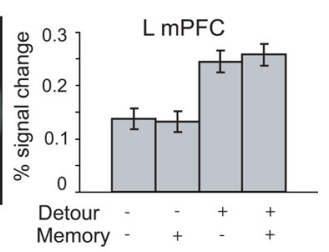

terior cingulate gyrus extending into the precuneus and retrosplenial cortex, cuneus, anterior cingulate and lateral temporal cortices, and the bilateral cerebellum (supplemental Fig. 2A; supplemental Table 1, available at www.jneurosci.org as supplemental material). These results were independent of the order of the regressors entered in the model. Hence, these regions are driven by goal proximity and not by the varying difficulty of the task (for effects of varying difficulty, see supplemental Fig. $2 B$, available at www. jneurosci.org as supplemental material). Note that percentage signal change for the control condition in the hippocampus is not positive and is lower than that for the shorter goal distances (Fig. 5), indicating that task ease at these shorter distances cannot account for hippocampal activation (since its activation is greater than for the control condition).

Could the effects of goal proximity reflect response-switching or novelty? Start position 6 had one of the longest path lengths to the goal and also involved choosing the opposite gate to that associated with the shortest path from the encoding viewpoint (middle position). It is therefore possible that an effect of goal proximity could arise in part from decreased activation in trials from start position 6, in which participants had to switch their response compared with any prepotent response selected during the encoding phase. No sign of this was found; the activation for start position 6 exceeded that for start position 4 , which was equidistant to the goal but did not require switching from a response selected during encoding (supplemental Fig. $3 A$, available at www.jneurosci.org as supplemental material). We also examined whether the goal proximity effect might be modulated by novelty, since the view from the different start positions involve differing amounts of change from the (middle) view shown at encoding. There was no sign of a parametric increase in activation according to the extent of visual change from encoding to test phases (supplemental Fig. 3B, available at www.jneurosci.org as supplemental material).

In the final analysis of goal proximity, we investigated those regions in which activation increased parametrically the further the start location was from the goal. These regions therefore showed increased activation when the comparison between the correct and incorrect routes was increasingly more difficult. The regions identified in this analysis included the precentral gyrus, bilateral insula, precuneus, dorsolateral and ventrolateral PFC, anterior and posterior cingulate cortices, angular gyrus, and PPC (Fig. 6; Table 4).

\section{Discussion}

We identified an extended network involved in spatial decision making in the absence of actual navigation. The most prominent activations were in the hippocampus, parahippocampal gyrus, $\mathrm{mPFC}, \mathrm{PPC}$, and precuneus. The $\mathrm{mPFC}$, precuneus, and PPC are more active when a detour must be planned, whereas the parahippocampal gyrus is more active when the layout of the scene 
must be remembered. A parametric analysis revealed increasing activation with the proximity of the goal in bilateral anterior hippocampus, right parahippocampal gyrus, $\mathrm{mPFC}$, and medial parietal regions, independent of task difficulty, response switching, and novelty. Previous studies have identified a similar network during active navigation in virtual environments (Maguire et al., 1998; Shelton and Gabrieli, 2002; Hartley et al., 2003; Iaria et al., 2003; Bohbot et al., 2004; Spiers and Maguire, 2006, 2007) and during imagined navigation (Ghaem et al., 1997). Here, we attempt to specify their functional contributions to spatial decision making.

\section{Detour planning}

Prefrontal involvement in spatial memory has been attributed variously to executive functions, planning, decision making, and working memory (Maguire et al., 1998; Grön et al., 2000; Hartley et al., 2003; Bohbot et al., 2004; Spiers and Maguire, 2006). Our results indicate that planning a detour activates the left mPFC and right ventromedial PFC, consistent with findings showing increased left mPFC activity for strategy switching in the presence of obstacles (Maguire et al., 1998; Rauchs et al., 2008) and right ventromedial PFC activation when a specific route is blocked (Spiers and Maguire, 2006). Our study allows further interpretation of these activations. First, they are not due to increased difficulty in the detour condition, since difficulty was controlled independently in our design, and did not produce MPFC activation. Second, mPFC activity is not a novelty response to the unexpected blockage, since detour trials were as common as nodetour trials in our design. Overall, these results implicate mPFC in changing strategy when encountering an obstacle, with inhibition of the most direct path likely an important component.

The precuneus and left PPC were activated by detour planning. Both regions are activated by way-finding compared with processing static scenes (Maguire et al., 1998) or route-following (Hartley et al., 2003). The PPC has also been associated with spatial planning (Spiers and Maguire, 2006) and activity there correlates with egocentric distance to the goal (Spiers and Maguire, 2007). Animal studies reveal neurons in medial parietal areas that respond to the position of stimuli in allocentric space (Galletti et al., 1993; Dean and Platt, 2006), whereas neurons in the more lateral intraparietal sulcus respond to combinations of egocentric and allocentric locations (Andersen et al., 1985; Snyder et al., 1998) and to the animal's position along a trajectory (Nitz, 2006). Thus, these activations may reflect a role in translating between allocentric medial temporal lobe representations and egocentric parietal representations necessary for imagery and planning of potential routes (Burgess et al., 2001; Byrne et al., 2007), which would be more complex in the detour condition.

\section{Effect of memory}

Memory-related activation was detected in bilateral parahippocampal and lingual gyri when the scene was obscured by a curtain compared with when it was visible. Parahippocampal and lingual activations have been detected during recall of topographic information (Aguirre et al., 1996), suggesting their role in learning to-
Table 2. Results of the detour $\times$ memory ANOVA

\begin{tabular}{|c|c|c|c|c|c|}
\hline \multirow[b]{2}{*}{ Region (BA) } & \multirow[b]{2}{*}{ Laterality } & \multicolumn{3}{|c|}{ MNI coordinates } & \multirow[b]{2}{*}{ Zscore } \\
\hline & & $x$ & $y$ & $z$ & \\
\hline \multicolumn{6}{|l|}{ No-detour $>$ detour } \\
\hline \multirow[t]{2}{*}{ Hippocampus } & $\mathrm{R}$ & 26 & -6 & -16 & 4.51 \\
\hline & $\mathrm{L}$ & -22 & -6 & -16 & $3.14^{*}$ \\
\hline \multicolumn{6}{|l|}{ Detour $>$ no-detour } \\
\hline \multirow[t]{2}{*}{ Medial PFC (6) } & $\mathrm{L}$ & -26 & -4 & 62 & 4.75 \\
\hline & $\mathrm{R}$ & 18 & 8 & 64 & 3.65 \\
\hline Medial PFC (10) & $\mathrm{L}$ & -18 & 58 & -8 & 3.55 \\
\hline Ventromedial PFC (9) & $\mathrm{R}$ & 20 & 38 & 26 & 3.47 \\
\hline \multirow[t]{2}{*}{ Precuneus (7) } & $\mathrm{R}$ & 2 & -60 & 58 & 4.69 \\
\hline & $\mathrm{L}$ & -4 & -52 & 52 & 3.86 \\
\hline PPC (40) & $\mathrm{L}$ & -56 & -44 & 50 & 3.59 \\
\hline \multirow[t]{2}{*}{ Cerebellum } & $\mathrm{R}$ & 34 & -46 & -38 & 4.77 \\
\hline & $\mathrm{L}$ & -42 & -52 & -46 & 3.72 \\
\hline \multicolumn{6}{|l|}{ No-memory $>$ memory } \\
\hline \multirow[t]{2}{*}{ Inferior occipital gyrus (18) } & $\mathrm{R}$ & 28 & -90 & -4 & 5.50 \\
\hline & $\mathrm{L}$ & -32 & -86 & -4 & 4.40 \\
\hline \multicolumn{6}{|l|}{ Memory $>$ no-memory } \\
\hline \multirow[t]{2}{*}{ Lingual gyrus (18/19) } & $\mathrm{R}$ & 6 & -78 & -2 & 4.96 \\
\hline & $\mathrm{L}$ & -6 & -80 & -6 & 4.74 \\
\hline \multirow[t]{2}{*}{ Parahippocampal gyrus (36) } & $\mathrm{R}$ & 28 & -38 & -18 & 3.89 \\
\hline & $\mathrm{L}$ & -22 & -46 & -6 & 3.33 \\
\hline \multicolumn{6}{|l|}{ Detour $\times$ memory interaction } \\
\hline \multirow[t]{2}{*}{ Ventrolateral PFC (45/47/44) } & $\mathrm{R}$ & 40 & 32 & 4 & 4.99 \\
\hline & $\mathrm{L}$ & -30 & 30 & -8 & 3.65 \\
\hline \multirow[t]{3}{*}{ Ventromedial PFC (9/8) } & $\mathrm{L}$ & -46 & 8 & 34 & 3.92 \\
\hline & $\mathrm{R}$ & 8 & 36 & 32 & 3.76 \\
\hline & $\mathrm{R}$ & 44 & 26 & 32 & 3.76 \\
\hline \multirow[t]{2}{*}{ Medial PFC/ACC (24/32) } & $\mathrm{R}$ & 8 & 10 & 34 & 3.67 \\
\hline & $\mathrm{R}$ & 10 & 24 & 38 & 3.41 \\
\hline Precentral gyrus (4) & $\mathrm{L}$ & -26 & -8 & 46 & 4.12 \\
\hline \multirow[t]{2}{*}{ Precuneus (7) } & $\mathrm{R}$ & 14 & -60 & 40 & 3.90 \\
\hline & $\mathrm{L}$ & -2 & -52 & 58 & 3.67 \\
\hline Hippocampus & $\mathrm{L}$ & -32 & -20 & -10 & 4.14 \\
\hline Fusiform gyrus (37) & $\mathrm{L}$ & -44 & -48 & -12 & 3.80 \\
\hline PPC (40) & $\mathrm{R}$ & 58 & -54 & 40 & 3.37 \\
\hline Cerebellum & $\mathrm{L}$ & -34 & -44 & -44 & 3.95 \\
\hline
\end{tabular}

${ }^{*} p<0.005, k>10$ voxels. ACC, Anterior cingulate cortex; BA, Brodmann area(s); L, left; $R$, right.
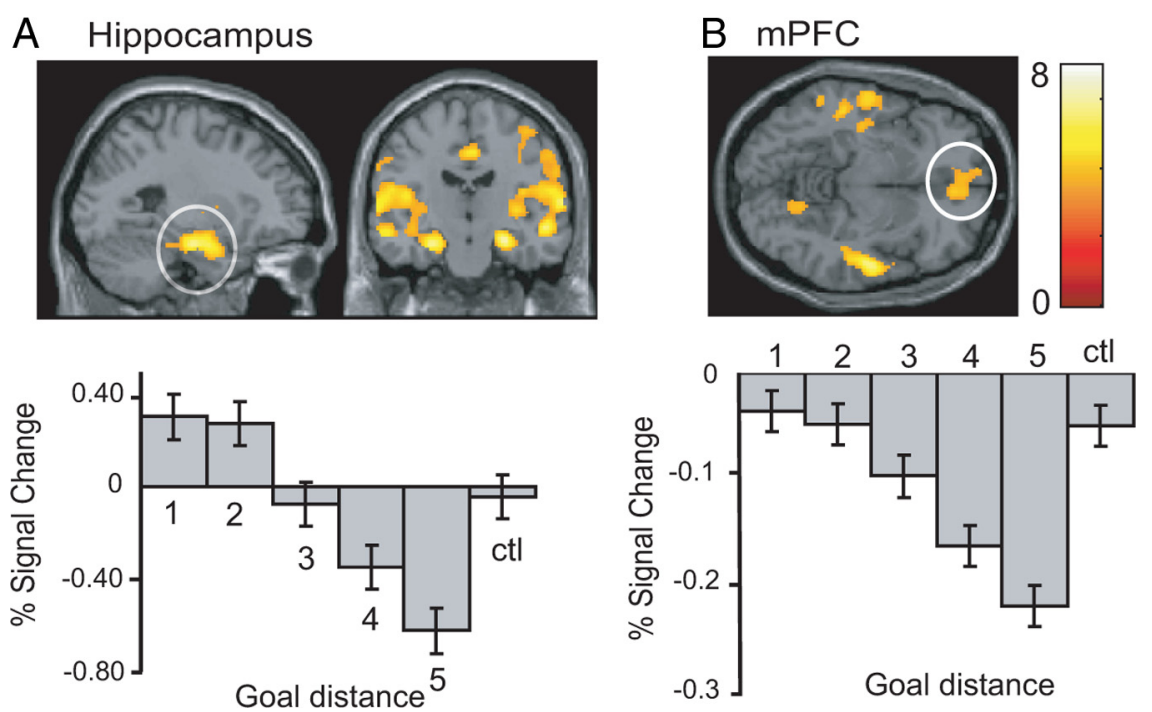

Figure 5. Parametric effect of goal proximity. $\boldsymbol{A}, \boldsymbol{B}$, Left hippocampal $(\boldsymbol{A} ; \boldsymbol{x}=-26, y=-16, z=-20)$ and left medial prefrontal $(\boldsymbol{B} ; \boldsymbol{x}=-2, y=56, z=-12)$ activation increases with goal proximity. Statistical parametric maps show the increasing response with increasing proximity to goal (top). Plots show activation ( \pm SEM) for the different levels of proximity to goal (Fig. 2) and control condition (ctl). Stereotaxic coordinates are given in Table 3. Color scale shows voxel $t$ values. For representational purposes, the statistical maps show clusters surviving a height threshold of $p<0.0001$. 
Table 3. Results of the parametric analysis of proximity to goal, showing regions more active for start positions closer to the goal

\begin{tabular}{|c|c|c|c|c|c|}
\hline \multirow[b]{2}{*}{ Region (BA) } & \multirow[b]{2}{*}{ Laterality } & \multicolumn{3}{|c|}{ MNI coordinates } & \multirow[b]{2}{*}{ Zscore } \\
\hline & & $x$ & $y$ & $z$ & \\
\hline Hippocampus & $\mathrm{R}$ & 24 & -12 & -18 & 6.81 \\
\hline Hippocampus & $\mathrm{L}$ & -26 & -16 & -20 & 5.86 \\
\hline Parahippocampal gyrus (35) & $\mathrm{R}$ & 22 & -4 & -24 & 6.17 \\
\hline \multirow[t]{4}{*}{ Ventromedial PFC (9/10/8) } & $\mathrm{L}$ & -10 & 56 & 28 & 4.67 \\
\hline & $\mathrm{R}$ & 8 & 62 & 22 & 4.67 \\
\hline & $\mathrm{L}$ & -8 & 46 & 42 & 4.84 \\
\hline & $\mathrm{L}$ & -12 & 36 & 48 & 4.66 \\
\hline \multirow[t]{2}{*}{$\operatorname{mPFC}(11 / 10)$} & $\mathrm{L}$ & -2 & 56 & -12 & 4.45 \\
\hline & $\mathrm{R}$ & 6 & 46 & -8 & 4.43 \\
\hline \multirow[t]{2}{*}{ Ventrolateral PFC (45/47) } & $\mathrm{L}$ & -50 & 30 & 4 & 3.83 \\
\hline & $\mathrm{L}$ & -40 & 32 & -14 & 3.39 \\
\hline \multirow[t]{2}{*}{ Precuneus/PCC/RSC (31/23/30) } & $\mathrm{L}$ & -2 & -38 & 32 & 5.74 \\
\hline & $\mathrm{R}$ & 4 & -48 & 28 & 5.49 \\
\hline Superior temporal gyrus (22) & $\mathrm{R}$ & 54 & -2 & 6 & 6.03 \\
\hline Superior temporal gyrus (22) & $\mathrm{L}$ & -60 & -40 & 18 & 5.97 \\
\hline Fusiform gyrus (37) & $\mathrm{R}$ & 36 & -52 & -16 & 3.75 \\
\hline Angular gyrus (39) & $\mathrm{L}$ & -58 & -56 & 16 & 6.49 \\
\hline SMA (6) & $\mathrm{R}$ & 10 & -10 & 70 & 3.75 \\
\hline \multirow[t]{2}{*}{ Lingual gyrus (19) } & $\mathrm{R}$ & 18 & -68 & -4 & 4.87 \\
\hline & $\mathrm{L}$ & -12 & -72 & -6 & 3.40 \\
\hline Cuneus (19) & $\mathrm{R}$ & 8 & -82 & 30 & 6.05 \\
\hline \multirow[t]{2}{*}{ Caudate nucleus } & $\mathrm{R}$ & 22 & -24 & 32 & 3.34 \\
\hline & $\mathrm{L}$ & -18 & -12 & 28 & 3.32 \\
\hline \multirow[t]{2}{*}{ Cerebellum } & $\mathrm{R}$ & 22 & -80 & -34 & 4.93 \\
\hline & $\mathrm{L}$ & -10 & -52 & -8 & 4.00 \\
\hline
\end{tabular}

$Z$ scores are for clusters of more than 10 voxels at $p<0.001$. BA, Brodmann area(s); RSC, retrosplenial cortex; SMA, supplementary motor area; $\mathrm{PCC}$, posterior cingulate cortex; $L$, left; $R$, right.
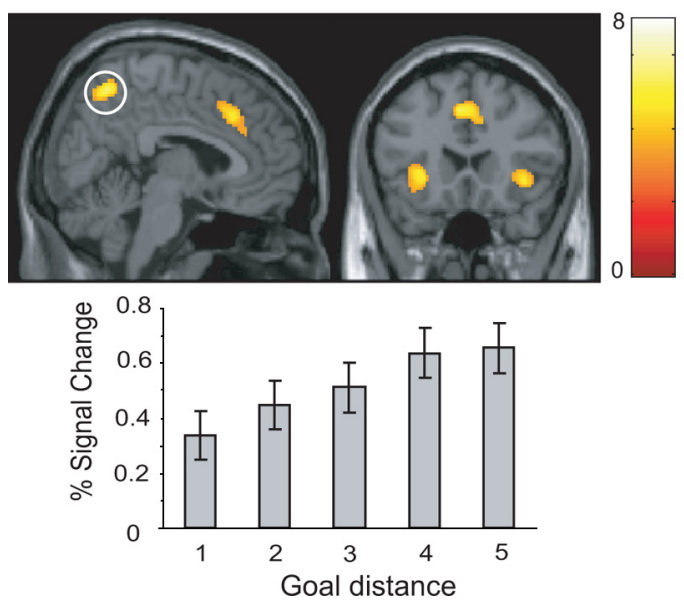

Figure 6. Parametric responses of increasing goal distance showing activation in the precuneus (sagittal section), insula (coronal section), and anterior cingulate cortex (both sections). Statistical parametric maps and plots of percentage signal change ( \pm SEM) in the left precuneus $(x=12, y=-58, z=60)$ are shown for increasing levels of distance from goal. The farther the start position is from the goal, the higher the activation. Stereotaxic coordinates are given in Table 4. Color scale shows voxel $t$ values.

pographical representations. The parahippocampal gyrus responds selectively to visual scenes depicting places (Epstein and Kanwisher, 1998) and is specifically involved in the retrieval of spatial context compared with nonspatial context (Burgess et al., 2001; King et al., 2005; Bar et al., 2008; Epstein and Ward, 2010). The absence of significant memory-related hippocampal activation is consistent with a long history of basic contrasts of memory versus nonmemory conditions (Ungerleider, 1995; Wagner et al., 1998). Our findings confirm the role of the parahippocampal gyrus in representing the spatial information in visual scenes
Table 4. Results of the parametric analysis of distance to goal, showing regions more active for start positions farther from the goal

\begin{tabular}{|c|c|c|c|c|c|}
\hline \multirow[b]{2}{*}{ Region (BA) } & \multirow[b]{2}{*}{ Laterality } & \multicolumn{3}{|c|}{ MNI coordinates } & \multirow[b]{2}{*}{ Zscore } \\
\hline & & $x$ & $y$ & $Z$ & \\
\hline Precentral gyrus (4) & $\mathrm{R}$ & 26 & 0 & 60 & 6.05 \\
\hline \multirow[t]{2}{*}{ Insula } & $\mathrm{L}$ & -30 & 24 & -2 & 4.76 \\
\hline & $\mathrm{R}$ & 34 & 26 & -2 & 4.48 \\
\hline \multirow[t]{2}{*}{ Precuneus (7) } & $\mathrm{R}$ & 12 & -58 & 60 & 5.75 \\
\hline & $\mathrm{L}$ & -16 & -62 & 60 & 5.47 \\
\hline Dorsolateral PFC (6) & $\mathrm{L}$ & -24 & 2 & 62 & 4.63 \\
\hline Ventrolateral PFC (44) & $\mathrm{R}$ & 50 & 10 & 22 & 4.24 \\
\hline $\mathrm{ACC}(32)$ & $\mathrm{L}$ & -4 & 22 & 42 & 4.85 \\
\hline$P C C(23)$ & $\mathrm{L}$ & -6 & 32 & 28 & 3.63 \\
\hline PPC (40) & $\mathrm{L}$ & -40 & -46 & 48 & 3.77 \\
\hline Cerebellum & $\mathrm{R}$ & 32 & -44 & -38 & 3.54 \\
\hline
\end{tabular}

$Z$ scores are for clusters of more than 10 voxels at $p<0.001$. BA, Brodmann area(s); ACC, anterior cingulate cortex $\mathrm{PCC}$, posterior cingulate cortex; $R$, right; $L$, left.

rather than just perceptual processing. In the reverse comparison, greater activation in bilateral posterior occipital regions reflected the richer visual characteristics of the scenes without the curtain.

\section{Effect of goal proximity}

The bilateral anterior hippocampus, right parahippocampal gyrus, medial prefrontal, and medial posterior parietal regions showed a very robust increase in activation with goal proximity. Activity in the anterior hippocampus can be interpreted in terms of complementary spatial and nonspatial processes. Models of rodent navigation posit that the place cell representation of location drives representations of reward expectation (Foster et al., 2000 ) or goal proximity (goal proximity and expectation of reward are equivalent in the context of navigation in simple environments) (Burgess et al., 1994; Burgess and O'Keefe, 1996; Bilkey and Clearwater, 2005). These goal cells would have firing fields covering the entire environment, with peak rates at the goal location, and were postulated for the subiculum or nucleus accumbens. However, the large firing fields in ventral rodent hippocampus (anterior hippocampus in humans) and the association of these regions with contextual, affective, and rewardrelated processing (Moser and Moser, 1998; Ferbinteanu and McDonald, 2001; Bast, 2007; Adhikari et al., 2010; Fanselow and Dong, 2010; Royer et al., 2010) suggest that the representation of goal proximity could be provided by the anterior hippocampus.

The ventral/anterior hippocampus is closely connected to medial prefrontal areas (Fanselow and Dong, 2010; Royer et al., 2010) where goal-responsive cells have been reported in rodents (Hok et al., 2005, 2007). Thus, anterior hippocampal and medial prefrontal areas may conspire to support representations of goal proximity (Gaussier et al., 2002; Killcross and Coutureau, 2003; Poucet et al., 2004; Doeller et al., 2008).

Our finding of hippocampal activation in spatial decision making is consistent with its association with navigating to an unseen target location (Maguire et al., 1998; Grön et al., 2000; Hartley et al., 2003; Iaria et al., 2003; Spiers and Maguire, 2006), and recordings in humans showing the presence of goalresponsive place cells in the hippocampus and prefrontal cortex (Ekstrom et al., 2003). In addition, hippocampal place cells can show a modulation of their spatially localized activity according to the goal location (Breese et al., 1989; Kobayashi et al., 1997; Hollup et al., 2001; Fyhn et al., 2002; Hok et al., 2007; Dupret et al., 2010) or current path (Frank et al., 2000; Ferbinteanu and Shapiro, 2003; Lee et al., 2006). Thus, our findings are consistent 
with the presence of a representation of goal proximity in the HF that is used for path planning.

The remaining parahippocampal activation presumably reflects support for the representation of the spatial scene within which planning is performed, consistent with its role in processing spatial scenes (Epstein and Kanwisher, 1998) and its activation in the memory condition. Medial parietal/posterior cingulate regions were also activated by goal proximity, although, unlike the hippocampus, these regions were also more active when planning detours (see Detour planning, above). Their activation may reflect the intensity of spatial processing and imagery, which might increase with both goal proximity and detour planning. This would be consistent with the association of medial parietal regions with spatial navigation (Burgess et al., 2002; Bird and Burgess, 2008; Epstein, 2008; Vann et al., 2009), episodic memory (Wagner et al., 2005; Viard et al., 2007, 2010, 2011), and imagery (Fletcher et al., 1995; Burgess et al., 2001; Cavanna and Trimble, 2006; Byrne et al., 2007). Studies in monkeys (McCoy and Platt, 2005) and humans (Peters and Büchel, 2009) have also linked these areas to reward processing and attention. Pearson et al. (2009) hypothesized that it integrates variables such as reward, uncertainty, and target location.

These findings indicate a role for the HF (including subiculum), medial prefrontal, and medial parietal regions in supporting goal-directed navigation, and suggest that this is achieved via increases in firing rate with goal proximity. Our results are consistent with the existence of this type of goal coding in hippocampal, parahippocampal, medial prefrontal, and medial parietal areas, and illustrate the potential for complementary spatial and nonspatial interpretations of anterior hippocampal processing.

A different network of regions was associated with increased distance from the goal, including lateral prefrontal, bilateral insula, anterior and posterior cingulate cortices, and lateral parietal regions. As distance increases, the two alternative routes become more similar and consequently the choice between them becomes more difficult. The network of regions identified by this analysis is consistent with previous associations of increasing dorsolateral prefrontal activation with task difficulty (Duncan and Owen, 2000) and anterior cingulate and ventrolateral prefrontal activation with task difficulty (Barch et al., 1997) and response selection (Tregellas et al., 2006).

\section{Conclusion}

Using a spatial route planning task, we identified an extensive network of brain regions showing specific involvement in detour planning, memory, and goal proximity. Activity in medial prefrontal cortex, precuneus, and left PPC was associated with detour planning, regardless of difficulty, whereas activity in the parahippocampal gyrus was associated with remembering the spatial layout of the scene. Activity in bilateral anterior hippocampus showed a robust increase with proximity to the goal, together with medial prefrontal and parietal areas. Our findings support results from single-unit recordings and computational modeling concerning the use of direct encoding of goal proximity in navigation, and highlight the potential for complementary spatial and nonspatial interpretations of anterior hippocampal function.

\section{References}

Adcock RA, Thangavel A, Whitfield-Gabrieli S, Knutson B, Gabrieli JD (2006) Reward-motivated learning: mesolimbic activation precedes memory formation. Neuron 50:507-517.

Adhikari A, Topiwala MA, Gordon JA (2010) Synchronized activity be- tween the ventral hippocampus and the medial prefrontal cortex during anxiety. Neuron 65:257-269.

Aguirre GK, Detre JA, Alsop DC, D’Esposito M (1996) The parahippocampus subserves topographical learning in man. Cereb Cortex 6:823-829.

Andersen RA, Essick GK, Seigel RM (1985) Encoding of spatial location by posterior parietal neurons. Science 230:456-458.

Bannerman DM, Rawlins JN, McHugh SB, Deacon RM, Yee BK, Bast T, Zhang WN, Pothuizen HH, Feldon J (2004) Regional dissociations within the hippocampus-memory and anxiety. Neurosci Biobehav Rev 28:273-283.

Bar M, Aminoff E, Schacter DL (2008) Scenes unseen: the parahippocampal cortex intrinsically subserves contextual associations, not scenes or places per se. J Neurosci 28:8539-8544.

Barch DM, Braver TS, Nystrom LE, Forman SD, Noll DC, Cohen JD (1997) Dissociating working memory from task difficulty in human prefrontal cortex. Neuropsychologia 35:1373-1380.

Bast T (2007) Toward an integrative perspective on hippocampal function: from the rapid encoding of experience to adaptive behavior. Rev Neurosci 18:253-281.

Bilkey DK, Clearwater JM (2005) The dynamic nature of spatial encoding in the hippocampus. Behav Neurosci 119:1533-1545.

Bird CM, Burgess N (2008) The hippocampus and memory: insights from spatial processing. Nat Rev Neurosci 9:182-194.

Bohbot VD, Iaria G, Petrides M (2004) Hippocampal function and spatial memory: evidence from functional neuroimaging in healthy participants and performance of patients with medial temporal lobe resections. Neuropsychology 18:418-425.

Breese CR, Hampson RE, Deadwyler SA (1989) Hippocampal place cells: stereotypy and plasticity. J Neurosci 9:1097-1111.

Bunzeck N, Düzel E (2006) Absolute coding of stimulus novelty in the human substantia nigra/VTA. Neuron 51:369-379.

Burgess N, O'Keefe J (1996) Neuronal computations underlying the firing of place cells and their role in navigation. Hippocampus 6:749-762.

Burgess N, Recce M, O'Keefe J (1994) A model of hippocampal function. Neural Netw 7:1065-1081.

Burgess N, Maguire EA, Spiers HJ, O’Keefe J (2001) A temporoparietal and prefrontal network for retrieving the spatial context of lifelike events. Neuroimage 14:439-453.

Burgess N, Maguire EA, O'Keefe J (2002) The human hippocampus and spatial and episodic memory. Neuron 35:625-641.

Byrne P, Becker S, Burgess N (2007) Remembering the past and imagining the future: a neural model of spatial memory and imagery. Psychol Rev 114:340-375.

Cavanna AE, Trimble MR (2006) The precuneus: a review of its functional anatomy and behavioural correlates. Brain 129:564-583.

Dayan P (1991) Navigating through temporal difference. In: Advances in neural information processing systems 3 (Lippman RP, Moody JE, Touretzky DS, eds), pp 464-470. San Mateo, CA: Morgan Kaufmann.

Dean HL, Platt ML (2006) Allocentric spatial referencing of neuronal activity in macaque posterior cingulate cortex. J Neurosci 26:1117-1127.

Doeller CF, King JA, Burgess N (2008) Parallel striatal and hippocampal systems for landmarks and boundaries in spatial memory. Proc Natl Acad Sci U S A 105:5915-5920.

Dudukovic NM, Wagner AD (2007) Goal-dependent modulation of declarative memory: neural correlates of temporal recency decisions and novelty detection. Neuropsychologia 45:2608-2620.

Duncan J, Owen AM (2000) Common regions of the human frontal lobe recruited by diverse cognitive demands. Trends Neurosci 23:475-483.

Dupret D, O’Neill J, Pleydell-Bouverie B, Csicsvari J (2010) The reorganization and reactivation of hippocampal maps predict spatial memory performance. Nat Neurosci 13:995-1002.

Eichenbaum H, Dudchenko P, Wood E, Shapiro M, Tanila H (1999) The hippocampus, memory, and place cells: is it spatial memory or a memory space? Neuron 23:209-226.

Ekstrom AD, Kahana MJ, Caplan JB, Fields TA, Isham EA, Newman EL, Fried I (2003) Cellular networks underlying human spatial navigation. Nature 425:184-188.

Epstein RA (2008) Parahippocampal and retrosplenial contributions to human spatial navigation. Trends Cogn Sci 12:388-396.

Epstein R, Kanwisher N (1998) A cortical representation of the local visual environment. Nature 392:598-601. 
Epstein RA, Ward EJ (2010) How reliable are visual context effects in the parahippocampal place area? Cereb Cortex 20:294-303.

Fanselow MS, Dong HW (2010) Are the dorsal and ventral hippocampus functionally distinct structures? Neuron 65:7-19.

Ferbinteanu J, McDonald RJ (2001) Dorsal/ventral hippocampus, fornix, and conditioned place preference. Hippocampus 11:187-200.

Ferbinteanu J, Shapiro ML (2003) Prospective and retrospective memory coding in the hippocampus. Neuron 40:1227-1239.

Fletcher PC, Frith CD, Baker SC, Shallice T, Frackowiak RS, Dolan RJ (1995) The mind's eye-precuneus activation in memory related imagery. Neuroimage 2:195-200.

Foster DJ, Morris RG, Dayan P (2000) A model of hippocampally dependent navigation, using the temporal difference learning rule. Hippocampus 10:1-16.

Frank LM, Brown EN, Wilson M (2000) Trajectory encoding in the hippocampus and entorhinal cortex. Neuron 27:169-178.

Fyhn M, Molden S, Hollup S, Moser MB, Moser E (2002) Hippocampal neurons responding to first-time dislocation of a target object. Neuron 35:555-566.

Galletti C, Battaglini PP, Fattori P (1993) Parietal neurons encoding spatial locations in craniotopic coordinates. Exp Brain Res 96:221-229.

Gaussier P, Revel A, Banquet JP, Babeau V (2002) From view cells and place cells to cognitive map learning: processing stages of the hippocampal system. Biol Cybern 86:15-28.

Ghaem O, Mellet E, Crivello F, Tzourio N, Mazoyer B, Berthoz A, Denis M (1997) Mental navigation along memorized routes activates the hippocampus, precuneus, and insula. Neuroreport 8:739-744.

Grön G, Wunderlich AP, Spitzer M, Tomczak R, Riepe MW (2000) Brain activation during human navigation: gender-different neural networks as substrate of performance. Nat Neurosci 3:404-408.

Hartley T, Maguire EA, Spiers HJ, Burgess N (2003) The well-worn route and the path less travelled: distinct neural bases of route following and wayfinding in humans. Neuron 37:877-888.

Hok V, Save E, Lenck-Santini PP, Poucet B (2005) Coding for spatial goals in the prelimbic/infralimbic area of the rat frontal cortex. Proc Natl Acad Sci U S A 102:4602-4607.

Hok V, Lenck-Santini PP, Roux S, Save E, Muller RU, Poucet B (2007) Goalrelated activity in hippocampal place cells. J Neurosci 27:472-482.

Hollup SA, Molden S, Donnett JG, Moser MB, Moser EI (2001) Accumulation of hippocampal place fields at the goal location in an annular watermaze task. J Neurosci 21:1635-1644.

Iaria G, Petrides M, Dagher A, Pike B, Bohbot VD (2003) Cognitive strategies dependent on the hippocampus and caudate nucleus in human navigation: variability and change with practice. J Neurosci 23:5945-5952.

Jung MW, Wiener SI, McNaughton BL (1994) Comparison of spatial firing characteristics of units in dorsal and ventral hippocampus of the rat. J Neurosci 14:7347-7356.

Killcross S, Coutureau E (2003) Coordination of actions and habits in the medial prefrontal cortex of rats. Cereb Cortex 13:400-408.

King JA, Hartley T, Spiers HJ, Maguire EA, Burgess N (2005) Anterior prefrontal involvement in episodic retrieval reflects contextual interference. Neuroimage 28:256-267.

Kjelstrup KB, Solstad T, Brun VH, Hafting T, Leutgeb S, Witter MP, Moser EI, Moser MB (2008) Finite scale of spatial representation in the hippocampus. Science 321:140-143.

Kobayashi T, Nishijo H, Fukuda M, Bures J, Ono T (1997) Task-dependent representations in rat hippocampal place neurons. J Neurophysiol 78:597-613.

Lee I, Griffin AL, Zilli EA, Eichenbaum H, Hasselmo ME (2006) Gradual translocation of spatial correlates of neuronal firing in the hippocampus toward prospective reward locations. Neuron 51:639-650.

Maguire EA, Burgess N, Donnett JG, Frackowiak RS, Frith CD, O'Keefe J (1998) Knowing where and getting there: a human navigation network. Science 280:921-924.

McCoy AN, Platt ML (2005) Expectations and outcomes: decision-making in the primate brain. J Comp Physiol A 191:201-211.

Moser EI, Kropff E, Moser MB (2008) Place cells, grid cells, and the brain's spatial representation system. Annu Rev Neurosci 31:69-89.

Moser MB, Moser EI (1998) Functional differentiation in the hippocampus. Hippocampus 8:608-619.

Nitz DA (2006) Tracking route progression in the posterior parietal cortex. Neuron 49:747-756.
O'Keefe J, Nadel L (1978) The hippocampus as a cognitive map. Oxford: Oxford UP.

Pearson JM, Hayden BY, Raghavachari S, Platt ML (2009) Neurons in posterior cingulate cortex signal exploratory decisions in a dynamic multioption choice task. Curr Biol 19:1532-1537.

Peters J, Büchel C (2009) Overlapping and distinct neural systems code for subjective value during intertemporal and risky decision making. J Neurosci 29:15727-15734.

Poucet B, Lenck-Santini PP, Hok V, Save E, Banquet JP, Gaussier P, Muller RU (2004) Spatial navigation and hippocampal place cell firing: the problem of goal encoding. Rev Neurosci 15:89-107.

Rauchs G, Orban P, Balteau E, Schmidt C, Degueldre C, Luxen A, Maquet P, Peigneux P (2008) Partially segregated neural networks for spatial and contextual memory in virtual navigation. Hippocampus 18:503-518.

Royer S, Sirota A, Patel J, Buzsáki G (2010) Distinct representations and theta dynamics in dorsal and ventral hippocampus. J Neurosci 30:17771787 .

Shelton AL, Gabrieli JD (2002) Neural correlates of encoding space from route and survey perspectives. J Neurosci 22:2711-2717.

Snyder LH, Grieve KL, Brotchie P, Andersen RA (1998) Separate body- and world-referenced representations of visual space in parietal cortex. Nature 394:887-891.

Spiers HJ, Maguire EA (2006) Thoughts, behaviour, and brain dynamics during navigation in the real world. Neuroimage 31:1826-1840.

Spiers HJ, Maguire EA (2007) A navigational guidance system in the human brain. Hippocampus 17:618-626.

Spiers HJ, Burgess N, Hartley T, Vargha-Khadem F, O’Keefe J (2001a) Bilateral hippocampal pathology impairs topographical and episodic memory but not visual pattern matching. Hippocampus 11:715-725.

Spiers HJ, Burgess N, Maguire EA, Baxendale SA, Hartley T, Thompson PJ, O'Keefe J (2001b) Unilateral temporal lobectomy patients show lateralized topographical and episodic memory deficits in a virtual town. Brain 124:2476-2489.

Squire LR (1992) Memory and the hippocampus: a synthesis from findings with rats, monkeys, and human. Psychol Rev 2:195-231.

Strange BA, Fletcher PC, Henson RN, Friston KJ, Dolan RJ (1999) Segregating the functions of human hippocampus. Proc Natl Acad Sci U S A 96:4034-4039.

Sutton RS, Barto AG (1998) Reinforcement learning: an introduction. Cambridge, MA: MIT.

Tregellas JR, Davalos DB, Rojas DC (2006) Effect of task difficulty on the functional anatomy of temporal processing. Neuroimage 32:307-315.

Ungerleider LG (1995) Functional brain imaging studies of cortical mechanisms for memory. Science 270:769-775.

Vann SD, Aggleton JP, Maguire EA (2009) What does the retrosplenial cortex do? Nat Rev Neurosci 10:792-802.

Viard A, Piolino P, Desgranges B, Chételat G, Lebreton K, Landeau B, Young A, De La Sayette V, Eustache F (2007) Hippocampal activation for autobiographical memories over the entire lifetime in healthy aged subjects: an fMRI study. Cereb Cortex 17:2453-2467.

Viard A, Lebreton K, Chételat G, Desgranges B, Landeau B, Young A, De La Sayette V, Eustache F, Piolino P (2010) Patterns of hippocampalneocortical interactions in the retrieval of episodic autobiographical memories across the entire life-span of aged adults. Hippocampus 20:153-165.

Viard A, Chételat G, Lebreton K, Desgranges B, Landeau B, de La Sayette V, Eustache F, Piolino P (2011) Mental time travel into the past and the future in healthy aged adults: an fMRI study. Brain Cogn 75:1-9.

Wagner AD, Schacter DL, Rotte M, Koutstaal W, Maril A, Dale AM, Rosen BR, Buckner RL (1998) Building memories: remembering and forgetting of verbal experiences as predicted by brain activity. Science 281:1188-1191

Wagner AD, Shannon BJ, Kahn I, Buckner RL (2005) Parietal lobe contributions to episodic memory retrieval. Trends Cogn Sci 9:445-453.

Weiskopf N, Hutton C, Josephs O, Deichmann R (2006) Optimal EPI parameters for reduction of susceptibility-induced BOLD sensitivity losses: a whole-brain analysis at $3 \mathrm{~T}$ and $1.5 \mathrm{~T}$. Neuroimage 33:493-504.

Wittmann BC, Schott BH, Guderian S, Frey JU, Heinze HJ, Düzel E (2005) Reward-related FMRI activation of dopaminergic midbrain is associated with enhanced hippocampus-dependent long-term memory formation. Neuron 45:459-467. 